\title{
The Evolution of Star Formation Rate Density of Galaxies
}

\author{
Joachim Wirsich \\ Independent Researcher, Berlin, Germany
}

Email address:

wirsich@cdsdesign.de

\section{To cite this article:}

Joachim Wirsich. The Evolution of Star Formation Rate Density of Galaxies. American Journal of Modern Physics. Vol. 10, No. 1, 2021, pp. 1-6. doi: 10.11648/j.ajmp.20211001.11

Received: October 30, 2020; Accepted: January 5, 2021; Published: February 20, 2021

\begin{abstract}
We present a semi-analytical calculation of the global star formation density (SFD) by using the well constrained cold dark matter (CDM) halo mass function. Both, halo masses $M_{H}(z)$ and stellar masses $M_{*}(z)$ are taken from observations of Ly $\alpha$ emitter (LAEs) and/or Lyman break galaxies (LBGs). Most of them, spectroscopically selected, are characterized by high star formation rates. The view of galaxy formation is mainly based on the hierarchical ("botton-up") cold dark matter model for structure formation. We have used the connection between the halo mass and the star formation rate in galaxies of the halo mass $M_{H}$ at redshift $z$. Our model has the advantage that we are able to calculate the global star formation rate $\rho_{*}(z)$ (in $\left.M_{\odot} y^{-1} M p c^{-3}\right)$ by a closed equation. All parameters $\left(M_{H}, M_{*}\right.$ and $\left.n\right)$ have a well-defined physical meaning. From the CDM spectrum, the power law index of the halo mass function is well constrained. Our results are compiled in Table 1 and Figure 1. Here our results are compared with observations and hydrodynamical simulations. The physical meaning of the evolution of comoving cosmic star density as a function of redshift with three epochs is discussed. We find a good agreement between the SFD inferred from observations and our model in the range of redshifts $z=0-7$.
\end{abstract}

Keywords: Galaxies: Evolution, Galaxies: High Redshift, Galaxies: Star Formation

\section{Introduction}

Galaxies formed at high redshifts play an important role in understanding about first stars and galaxies lighting up in an expanding Universe. E.g. observations at redshift $z \sim 6$ corresponds to $90 \%$ of the age of the Universe. The redshifts of galaxies are inferred either through a search for Lyman-emission lines (Lyman $\alpha$ emitter) (LAEs) or through a search for a spectral break associated with the absorption of intervening hydrogen (Lyman break galaxies) (LBGs) [1, 2]. The LAEs at high redshift are viewed as being main progenitors of our Galaxy. Large Ly $\alpha$ blobs with filamentary structure have halos with a mass of $M_{H} \approx 10^{12} M_{\odot}$ at redshift $z \sim 2$ - 3 [3]. The LBGs are not the first generation objects, the LBGs having experienced metal enrichment from previous stellar generation [4].

Galaxy candidates for the first galaxies were identified by deep optical/near infrared surveys up to redshifts of $z \sim 10$ (450 million years after the Big Bang) [5]. Besides: already in 2010 the SDSS has discovered 20 bright AGNs around $z \sim 6$ ( $\sim$ 1Gyr after Big Bang). They reside in massive black holes $M_{B H} \approx 10^{9} M_{\odot}[6]$.
Theoretical studies suggest that very massive stars with $M_{*} \geq 100 M_{\odot}$ may be formed in primordial subhalos with mass lower than $M_{H} \sim 10^{6} M_{\odot}$ [7]. Detection of galaxies in the early Universe at high redshifts will allow some answers as to how reionization occurred. How the primordial gas was ionized is one of the most important questions in modern cosmology.

We assume that the process of reionizing hydrogen ends at $z \sim 6$ [8]. Indications for this are given by observations of the Ly $\alpha$ forest at $z \sim 6$ [9]. The SFD $\rho_{*}(z)$ is defined as the mass of new stars unit and comoving volume (in $M_{\odot} y^{-1} M p c^{-3}$ ). As $\rho_{*}(z)$ is a function of redshift $z$, it controls how many stars are formed in the course of time. E.g. investigations of SFD in galaxies study the early Universe and stars formed efficiency at a very early time. The dependence of $\rho_{*}(z)$ with redshift is called "Madau plot", because these authors have published the SFD for the first time [10].

The quality of our paper is that the SFD is calculated analytical by a well-defined close equation (see equation (7) below). This paper is organized as follows: in section 2 , we describe a model of the star formation history in cold dark 
matter (CDM) halos. In section 3, we present the results and in section 4 the discussion. In section 5 we summarize our results.

\section{Star Formation History of Galaxies in CDM-Halos}

In 1976, Brent Tully and Rich Fisher found a tight correlation between the luminosity of the disk of a galaxy and the rotation velocity: the luminosity is proportional to the mass of the stellar disk [11]. On the other hand, the rotation velocity is set up by the cold dark matter in the halo of the galaxy. The Tully-Fisher relation is important for understanding of connection of star formation in galaxies and the dynamical mass due to the CDM: Bouché et al. have shown the strong relation of the Tully-Fisher relation and the star formation galaxies (SFGs) [12]. E.g. their equation (15) demonstrates stellar fraction $f_{*}$ in galaxies in dependence of the total halo mass $M_{H}$ (in time evolution between the redshift $z \sim 2$ and today).

Mo, van den Bosch \& White show in their Figure 2.35 a modern Madau-plot when using measurements of LAEs and LBGs [13]. Already in 2011 over 100 LAEs for $z \sim 6$ have been photometrically selected and spectroscopically identified [14]. Let us consider the connection between the halo mass and the star formation history by using

$$
\rho_{*}(z)=\int\left\langle\dot{M}_{*}\left(M_{H}, z\right)\right\rangle n\left(M_{H}, z\right) d M_{H},
$$

where $\left\langle\dot{M}_{*}\left(M_{H}, z\right)\right\rangle$ is the mean star forming rate in the central galaxies of the halo mass $M_{H}$ at redshift $z$ [13 (see section 15.4.2 therein)]. $n\left(M_{H}, z\right)$ is the mass function of the dark matter at redshift $z$ [13 (see section 15.3 therein), 15 (16.3 therein)]. $n\left(M_{H}, z\right)$ can be approximated by

$$
\begin{gathered}
n\left(M_{H}, z\right)=\frac{1}{2 \sqrt{\pi}}\left(1+\frac{n}{3}\right) \frac{\bar{\rho}}{M_{H}^{2}}\left(\frac{M_{H}}{\bar{M}_{H}}\right)^{\frac{3+n}{6}} \\
\exp \left[-\left(\frac{M_{H}}{\bar{M}_{H}}\right)^{\frac{3+n}{3}}\right],
\end{gathered}
$$

where for a CDM spectrum the power law index ("spectral index") scales over a range of $-3<n<1[15,13$ (see section 5.6 therein)]. $\bar{\rho}$ is the average comoving density of the Universe (see below). $\bar{M}_{H}(z)$ is the characteristic halo mass at redshift $z$ via

$$
\bar{M}_{H}=\bar{M}_{H, 0}(1+z)^{-3},
$$

where the value of $n=-1$ was used [16 (section 7.5.2 therein)].

The star formation rate in the halo mass can be written as

$$
\dot{M}_{*}(z)=M_{*} H(z)=M_{*} H_{0} \Omega^{1 / 2}(1+z)^{3 / 2},
$$

[17 (equation 5.60)].

$\bar{\rho}$ is given by $\rho_{0}=1.88 \times 10^{-29} \Omega h^{2}\left(\right.$ in $\left.g \times \mathrm{cm}^{-3}\right)([17$ (section 5 therein)]. ( $\Omega=1$, [16 (see Table 8.2 therein)]. $H_{0}$ is the Hubble parameter at the present time and the reduzed Hubble parameter $h$ is defined as

$$
h=\frac{H_{0}}{100 k m s^{-1} M p c^{-1}} .
$$

Data of the halo mass $\bar{M}_{H, 0}$ are rare: Schneider report values from observations of LBGs as $3 \times 10^{11} M_{\odot}$ at $z=3$ and $10^{12} M_{\odot}$ at $z=2$, respectively [16 (section 9.1)]. Mo, van den Bosch \& White favor a value of $5 \times 10^{11} M_{\odot} h^{-1}[13$ (see section 7.3 therein)]. The characteristic mass increases with time: thus, as time passes more and more massive halos will start to form [13]. We choose a fixed value of $\bar{M}_{H, 0}=$ $10^{12} M_{\odot}$. Putting everything together we find $(\Omega=1)$

$$
\rho_{*}(z)=\frac{1.88 \times 10^{-29}}{2 \sqrt{\pi}} h^{2} H_{0}(1+z)^{3 / 2} \frac{2}{3} M_{*} \int_{M_{\min }}^{M_{\max }} \frac{1}{M_{H}^{2}}\left(\frac{M_{H}}{\bar{M}_{H}}\right)^{1 / 3} \exp \left[-\left(\frac{M_{H}}{\bar{M}_{H}}\right)^{2 / 3}\right] \mathrm{d} M_{H} .
$$

We set $\left(\frac{M_{H}}{\bar{M}_{H}}\right)^{1 / 3}=\nu$ and $\mathrm{d} M_{H}=\bar{M}_{H} 3 \nu^{2} \mathrm{~d} \nu$. Then the integral forms as

$$
\int_{\nu_{\min }}^{\nu_{\max }} \frac{1}{M_{H}^{2}} \nu \bar{M}_{H} 3 \nu^{2} \exp \left(-\nu^{2}\right) \mathrm{d} \nu
$$

By using (3) we find for $\rho_{*}(z)$ (in $\left.g \times \mathrm{cm}^{-3} \times s^{-1}\right)$ the result

$$
\rho_{*}(z)=\frac{1.88 \times 10^{-29}}{2 \sqrt{\pi}} h^{2} H_{0}(1+z)^{3 / 2} \frac{2}{3} \frac{\bar{M}_{H, 0} M_{*}}{M_{H}^{2}} \frac{3}{(1+z)^{3}} \int_{\nu_{\min }}^{\nu_{\max }} \nu^{3} \exp \left(-\nu^{2}\right) \mathrm{d} \nu .
$$

As Mo, van den Bosch \& White we assume that the ratio $\frac{M_{H}}{M_{H}}$ is independent of redshift $z$ and write with $\nu=\left(\frac{M_{H}}{M_{H}}\right)^{1 / 3}$

$$
\int \nu^{3} \exp \left(-\nu^{2}\right) \mathrm{d} \nu \approx \frac{M_{H}}{\bar{M}_{H}} \int_{\nu_{\min }}^{\nu_{\max }} \exp \left(-\nu^{2}\right) \mathrm{d} \nu \approx \int_{1}^{\infty} \exp \left(-\nu^{2}\right) \mathrm{d} \nu \approx \frac{\sqrt{\pi}}{2} .
$$


[13, 18 (fourth edition, equation $\mathrm{BI}(29)$ )]. Equation (7) present a semi-analytical model that contains reliable data as shown in Table 1 .

\section{Results}

Our results are compiled in Table 1 and Figure 1. The second row in Table 1 contains halo mass $M_{H}\left(M_{\odot}\right)$ in dependence of redshift $z$. The third row shows the reference for these. E.g. relates the value of the halo mass of $5 \times 10^{11} M_{\odot}$. at $z=6$ from LAEs as observed by Ouchi et. al. [19]. The smallest halo mass in Table 1 is $5 \times 10^{11} M_{\odot}$ : this is in the range of hydrodynamical simulations of Pillipich et. al. in their runs of "Illustris" and "TNG", the authors found as smallest halo masses of $10^{11} M_{\odot}$ and $10^{12} M_{\odot}$, respectively [20].

The data in the fourth row are the stellar mass $M_{*}\left(M_{\odot}\right)$ with references below. The majority of LBGs have stellar mass of $M_{*} \sim 10^{10} M_{\odot}$ of $z=3$ to $z=6$. The stellar masses in Table 1 are in the range of $5 \times 10^{9}-10^{10} M_{\odot}$ between $z=6$ and $z=4$ : the stellar mass $M_{*}$ of LBGs evolve in the range of $z=5$ to $z=4$ marginally only as shown by Stark et. al. [44, 23].

Table 1. The global star-formation rate $\rho_{*}(z)\left(\right.$ in $\left.M_{\odot} y^{-1} M p c^{-3}\right)$.

\begin{tabular}{|c|c|c|c|c|c|c|c|c|c|}
\hline $\mathrm{z}$ & 7 & 6 & 5 & 4 & 3 & 2.5 & 2 & 1 & 0 \\
\hline$M_{H}\left(M_{\odot}\right)$ & $5 \times 10^{11}$ & $5 \times 10^{11}$ & $5 \times 10^{11}$ & $5 \times 10^{11}$ & $10^{12}$ & $10^{12}$ & $10^{12}$ & $10^{13}$ & $10^{13}$ \\
\hline Ref. & [19] & [19] & [19] & {$[12,19]$} & {$[37,19,12]$} & {$[37,26,21]$} & {$[37,3,21]$} & {$[50]$} & {$[50]$} \\
\hline$M_{*}\left(M_{\odot}\right)$ & $5 \times 10^{9}$ & $5 \times 10^{9}$ & $10^{10}$ & $10^{10}$ & $10^{11}$ & $10^{11}$ & $10^{11}$ & $10^{11}$ & $5 \times 10^{9}$ \\
\hline Ref. & {$[38,21]$} & {$[46,45,23]$} & [46] & {$[12,47]$} & {$[48,49,47]$} & [49] & {$[25,47]$} & {$[28,27]$} & see text \\
\hline$\rho_{*}(z)$ & $4.3 \times 10^{-3}$ & $5.2 \times 10^{-3}$ & $1.4 \times 10^{-2}$ & $1.2 \times 10^{-1}$ & $2.5 \times 10^{-1}$ & 0.15 & 0.19 & 0.04 & $3.3 \times 10^{-3}$ \\
\hline
\end{tabular}

Analyses of star populations show that the LBGs at $z \sim 5$ have less massive galaxies than comparable samples of $z \sim 3$ [24]. Studies of optically selected galaxies, using the BM/BX method at $z \sim 2$, show stellar masses in the range of $10^{11} M_{\odot}$ [25]. They reside in dark matter halos of $M_{H} \approx 2 \times 10^{12} M_{\odot}$. Steidel et al. report a halo mass of $10^{12} M_{\odot}$ at $z=2.5$ [26]. The value of $M_{*}=10^{11} M_{\odot}$ at the redshift $z=1$ is taken from Noeske et al. [27, 28].

Let us calculate the global SFD at redshift $z=3$ and $z=0$, respectively. For $z=3$, we find, using equation (7) and the data from Table 1 (in $M_{\odot} y^{-1} M p c^{-3}$ )

$$
\rho_{*}(z=3) \approx \frac{1.88}{2 \sqrt{\pi}} h^{2} 720.7510^{-2} \frac{\sqrt{\pi}}{2} \approx 0.25 h^{2},
$$

where $H_{0}=72 \mathrm{~km} \mathrm{~s}^{-1} \mathrm{Mpc}^{-1}$ and the fixed value of $\bar{M}_{H, 0}=10^{12} M_{\odot}$ were used.

The star formation rate (SFR) for early type galaxies at redshift $z=0$ are poorly known. Spaans \& Carollo present volume-averaged SFRs for three galaxies of Hubbletype E0 and E2, the authors found values of SFDs lower than $10^{-2} M_{\odot} y^{-1} M p c^{-3}$ [29]. The SFRs are lower than $0.1 M_{\odot} y^{-1}$ at $z=0$. The early-type galaxy E4 shows a SFR of $1 M_{\odot} y^{-1}$ and a volume-averaged star formation rate of $0.1 M_{\odot} y^{-1} M p c^{-3}$ [29]. Treu, Möller \& Bertin studied a sample of 30 field E/S0 galaxies up to redshifts of $z=0.66$. Several of them show a significant emission of line [OII] $\lambda 3727$. If attributed to star formation, the line can correspond to SFRs of $0.5-5 M_{\odot} y^{-1}$ [30]. Li et. al. report for the edgeon S0 galaxy NGC 5866 $(d=11.21 M p c)$ a SFR lower than $0.1 M_{\odot} y^{-1}$ [31]. The stellar mass is $3 \times 10^{9} M_{\odot}$.

Using the Eddington time of $t_{E}=4.4 \times l 0^{8} y$ and a SFR of $0.5 M_{\odot} y^{-1}$, we find a stellar mass of $2 \times 10^{8} M_{\odot}$ at $z=0$
[13]. In total we obtain the final result

$$
\rho_{*}(z=0) \approx 2 \times 10^{-4} M_{\odot} y^{-1} M p c^{-3} .
$$

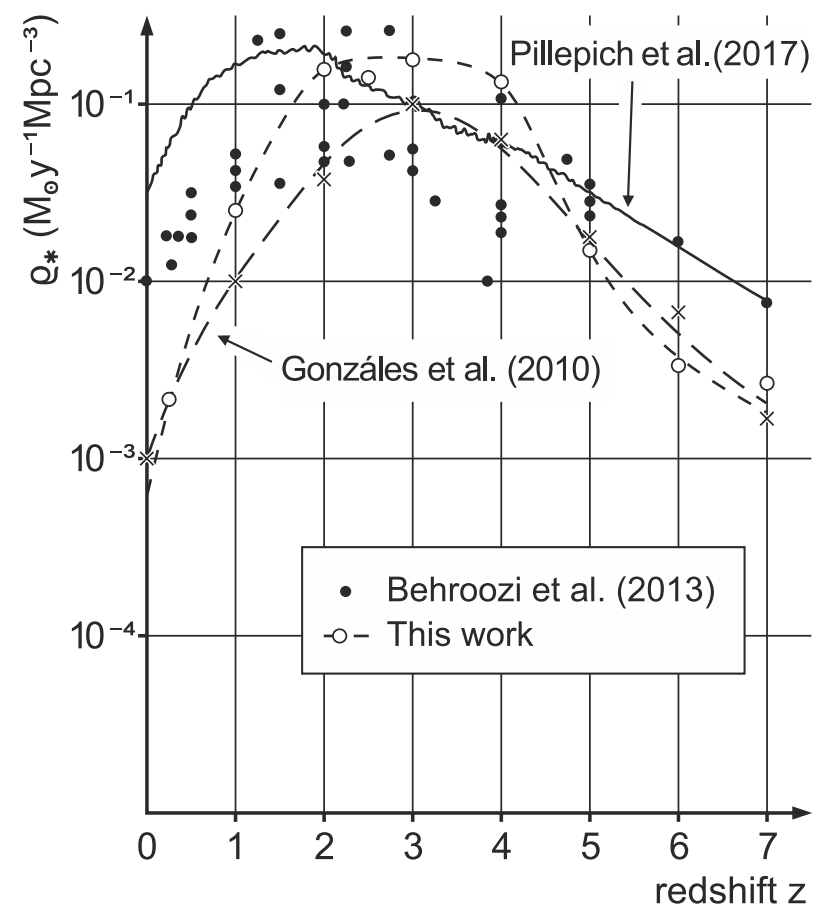

Figure 1. Star formation rate density as a function of redshift $z$ : Filled dots represent observations from Behroozi et. al. (see their Fig. 5 and Table 4) [21]. Crosses are from observations from Gonzáles [22]. The solid line is from Pillepich et. al. (see their Fig. 9) [20]. Open circles are from this work. 
On the other hand, one can do better: Overzier et. al. have observed local LGBs in the range of $z=0.2-0.5$ [32]. They conclude that the galaxies are similar to those LGBs at high redshifts. E.g. the stellar masses are in the range of $M_{*} \sim 5 \times 10^{9} M_{\odot}$. Using the halo mass of $M_{H} \sim 10^{13} M_{\odot}$ and $M_{*} \sim 5 \times 10^{9} M_{\odot}$ at $z=0.2$, we find the result (in $\left.M_{\odot} y^{-1} M p c^{-3}\right)$

$$
\rho_{*}(z=0.2) \approx 3.3 \times 10^{-3} h^{2} .
$$

Our results are compiled in Table 1 and Figure 1. Here the observations from Behroozi, Wechsler \& conroy and Gonzáles et al. are shown as well, respectively (error bars neglected) [21, 22]. The results of Pillepich et. al. are also shown [20]. Behroozi et al. report at $z=7$ a result of $\rho_{*} \approx 8 \times$ $10^{-3} M_{\odot} y^{-1} M p c^{-3}$ (Figure 1 below therein) [21]. Compared with our results from Table 1, we find that the value of $4.3 \times 10^{-3} M_{\odot} y^{-1} M p c^{-3}$ at $z=7$ is not far from the value given above.

Gonzáles et al. present in their Table 3 values of the stellar mass at $z \sim 7$ in the range of $M_{*} \approx 2 \times 10^{8}-1.2 \times 10^{10} M_{\odot}$ with a best fit of $M_{*} \approx 6.3 \times 10^{9} M_{\odot}$ at $z \sim 7$ when using the drop-out technique [22]. For the SFD Gonzáles et al. report a value of $8 \times 10^{-3} M_{\odot} y^{-1} M p c^{-3}$ at $z \sim 8$ (see their section 7.3) [22].

Oesch et al. found a peak in the evolution of star formation density at $z \sim 2.5$ with a numerical value of $\sim 3.2 \times$ $10^{-2} M_{\odot} y^{-1} M p c^{-3}$ (from Figure 15 therein) [33]. We find at $z \sim 2.5$ a result of $1.5 \times 10^{-1} M_{\odot} y^{-1} M p c^{-3}$ (see Table 1). Bouwens et al. report ultra-deep Advanced Camera for Surveys (ACS) and WFC3/IR observations of galaxies from $z \sim 7$ to $z \sim 4$. They found a SFD with a peak around $z \sim 3$ (see their Figure 19 and Figure 20 therein) with the result of $10^{-1} M_{\odot} y^{-1} M p c^{-3}$ [34]. The numerical values of the SFDs given in Table 7 are close to our results of Table 1: we find values of $1.2 \times 10^{-2}-4.3 \times 10^{-3} M_{\odot} y^{-1} M p c^{-3}$ in the range of $z=4-7$. We find a peak of the SFD at $z=2$ with $1.9 \times 10^{-1} M_{\odot} y^{-1} M p c^{-3}$ (Table 1). This is in the range results from Reddy et al., where star forming galaxies at $z \sim 2$ are selected by the $\mathrm{U}_{\mathrm{n}} \mathrm{GR}$ color and BzK colors, respectively [25].

The physical meaning of the peak at $z=2-3$ is that the efficiency of the baryon accretion is highest for halos in the range of $M_{H} \approx 10^{12} M_{\odot}$. The evolution of the comoving cosmic star formation density as a function of redshift show three main epochs [35]. The first epoch represents the epoch from cosmic reionization at $z \sim 10$ to $z \sim 6$ when first light from first galaxies and AGNs reionize the neutral IGM that permeate the Universe [8]. The next epoch is the epoch where the cosmic star formation rate peaks around $z=2-3$. Below the halo mass of $10^{12} M_{\odot}$, the accretion of cold gas is suppressed in the range of $z \sim 2-6$. The origin for this is the photoionizing in connection with the hard UV-radiation after reionization. At $z \sim 2$, the star formation rate is favoured by high accretion rates of cold baryon gas. The third epoch is the decline of cosmic star formation rate density from $z \sim 2$ down to the present at $z=0$. One of the reasons for the whole behaviour of $\rho_{*}(z)$ may be the idea that the Gaussian random field of CDM pertubations reflects itself in $\rho_{*}(z)$ [15 (section 16.3)].

The feedback induced by supernovae explosions heat the gas from the cold to the hot phase. The counterpart halo mass which supports the formation of stellar mass is $10^{11}-10^{12} M_{\odot}$. The adequate stellar mass is $10^{10}-10^{11} M_{\odot}$. (Table 1). The supernovae heat the interstellar medium and the gas is driven out of the galaxy. But the supernovae are not very efficient in transforming the stellar mass into e.g. kinetic energies: the efficiency is $f_{S N}=E_{S N} / 100 M_{\odot} c^{2}$ where $E_{S N}$ means the kinetic energy of ejects from the supernovae (in $10^{51} \mathrm{erg}$ ): $f_{S N} \sim 5.6 \times 10^{-6}$. Further, galactic winds play a minor role as a mechanism of feedback, since they would wash out the abundance gradients of galaxies. Galactic winds would change the $[\alpha / F e]$ versus $[\mathrm{Fe} / \mathrm{H}]$-relation in galaxies. Abundances gradients are derived from HII-regions in external galaxies. Radial gradients of e.g. $[O / H]$ versus $R / R_{25}$ are a common feature of spiral disk [36].

\section{Discussion}

Let us present some comments about the reliability of the data given in Table 1:

Adelberger et al. study galaxies in the BM, BX and LBG samples for redshifts $z=1.77, z=2.32$ and $z=2.97$, respectively. By using the angular correlation technique for virial dark matter halos, they found that the agreement is best if galaxies in the BM, BX and LBG samples are associated with halos of mass $M_{H} \sim 10^{12.1}, 10^{12.0}$ and $10^{11.5} M_{\odot}$, respectively [37].

Labbé state that the average uncertainties of stellar masses are approximately a factor $2-3$ (in the range of $z_{p h} \approx 6.8$ 7.2) [38].

Reddy \& Steidel conclude that the bulk of BX/LBG have stellar masses in the range of $10^{9}-10^{11} M_{\odot}(z=3-2)$. Stellar masses of $M_{*}>10^{11} M_{\odot}$ comprise with a very little fraction of $\leq 2 \%$ of all UV-faint galaxies [49]. Gonzáles et al. found that the estimated stellar masses for indivdual $z \sim 7 z$ dropouts in their sample range from $0.2 \times 10^{9}-12 \times 10^{9} M_{\odot}$. The estimated masses are much more well-constrained then other quantities - like the age - but nevertheless still uncertain at the factor of 2 level (see section 6 and Table 3 therein) [22].

Ouchi et al. found that the average hosting dark halo masses of LAEs are about $10^{11 \pm 1} M_{\odot}$ over $z=2-7$ (section 4.3 therein). On the other hand, a typical halo mass of LBG is estimated to be $10^{12 \pm 1} M_{\odot}$ about one order of magnitude larger than that of LAEs [19 (section 6.2 therein)].

Let us compare our results with some observations based on data of Hopkins [39]. Mo, van den Bosch \& White show in their Figure 2.35 the global star formation rate density as a function of redshift $z$ [13].

The figure has a peak of around $z \sim 2.7$, where $\rho_{*} \sim$ $10^{-0.5} M_{\odot} y^{-1} M p c^{-3}$. $\rho_{*}$ drops by an order of magnitude from $z \sim 2$ to the present. Ciardullo et al. report data of SFDs for redshift $z=2-4$ based on $\operatorname{Ly} \alpha$ emitter measurements 
[40]. Further, Harrison et al. show in their Figure 1 the volume averaged star formation rate density at redshift $z=0-3.5$ due to observations by optical and K-band surveys on Very Large Telescope (VLT): here it was shown that both the star formation and the activity of AGNs have a peak during the epoch of $z=1$ - 3 [41]. Recently, surveys by ALMA have published the dust-obscured star formation rate in the range of redshift $z=0-8$ [35]. Dunlop concludes that in the epoch of $z=2-2.5$, the star formation rate is dominated by dustobscured emission from massive galaxies. On the other hand, beyond $z=4$, the SFD is governed by unobscured emission from galaxies [35].

Behroozi, Wechsler \& Conroy present a five-parameter model. They found in their best-fitting model that the halo mass of $10^{12} M_{\odot}$ is most efficient for formation stars at all redshifts. In Figure 3, Behroozi et al. show their results compare to observations. The latter show a peak at $0.1 M_{\odot} y^{-1} M p c^{-3}$ at $z \sim 2$ as Dunlop [35]. The best-fit model of Behroozi et al. shows a drop of SFD after $z \sim 2.5$, which means a downturn of the baryon conversion efficiency [21].

Our model has the advantage that we are able to calculate $\rho_{*}(z)$ by a closed equation (see equation (7)). All parameters $\left(M_{H}, M_{*}\right.$ and $n$ ) have a well-defined physical meaning. In contrast to this: Due to the unknown nature of the CDM, Pillepich et al. are forced to use softening parameter (softening length $\varepsilon$ ) to prevent numerical divergences when two particles pass close to one another [20]. Further, the softening is necessary to prevent computer investment in eliminating the effects of close encounters, because the real physical system is completely collisionless. The softening parameter have the serious shortcoming of no any physical meaning.

\section{Conclusion}

The view of galaxy formation is mainly based on the hierarchical ("botton-up") cold dark matter mode. Therefore, we have used the connection between the halo mass and the star formation rate in galaxies of the halo mass $M_{H}$ at the redshift $z$. The data of halo masses and stellar masses are from observations of LAEs and/or LBGs up to redshifts of $z=7$. By using a closed equation which contains the halo mass function $n\left(M_{H}, z\right)$, we are able to describe analytical the global star formation rate density of galaxies from $z=0$ to $z=7$ (see equation (7)).

Our results are compiled in Table 1 and Figure 1. Here our results are compared with observations and hydrodynamical simulations. All parameters $\left(M_{H}, M_{*}\right.$ and the power law index $n$ ) have a well-defined physical meaning. The cold matter model follow the botton-up scenario: Our Galaxy may be a product of such a scenario, because it shows old stars and was formed at redshift $z \sim 2$. $M_{H}$ (Milky Way) = $1.0_{-0.1}^{+0.3} \times 10^{12} M_{\odot}$ at $r_{v i r}=275 k p c$ and $c=6.6_{-1.5}^{+1.2}$ [42]. Typical masses of $M_{H}$ for nearby Sbc galaxies are $3.60 \times 10^{11}$ $-9.68 \times 10^{11} M_{\odot}[43$ (Table 1)].

\section{Acknowledgements}

The author is grateful to an anonymous referee for critical comments which improved the manuscript.

The author is grateful to Bernhard Steuber for patient encouragement in preparation of the paper.

\section{References}

[1] Kashikawa, N., Shimasku, K., Matsuda, Y. et al. 2011, ApJ, 734, 119.

[2] Steidel, C. C., Shapley, A. E., Pettini, M. et al. 2004, ApJ, 604,534 .

[3] Yajima, H., Li, Y., Zhu, Q. et al. 2012, ApJ, 754, 118.

[4] Papovich, C., Dickinson, M. \& Ferguson, H. C. 2001, ApJ, 559, 620.

[5] Stark, D. P. 2016, ARA \& A, 54, 761.

[6] De Rosa, G., Decarli, R., Walter, F. et al. 2011, ApJ, 739, 56.

[7] O’Shea, B. W. \& Norman, M. L. 2007, ApJ, 654, 66.

[8] Fan, X., Carilli, C. \& Keating, R. 2006, ARAA; 44, 415.

[9] Becker, G. D., Rauch, M. \& Sargent, W. L. W. 2007, ApJ, 662,72 .

[10] Madau, P., Ferguson, H. C., Dickinson, M. et al. 1996, MNRAS, 283, 1388.

[11] Tully, B. \& Fischer, R. 1977, A \& A, 54, 661.

[12] Bouché, N., Dekel, A., Genzel, R. et al. 2010, ApJ, 718, 1001.

[13] Mo, H., van den Bosch, F. \& White, S. 2011, Galaxy Formation and Evolution (Cambridge Univ. Press, Cambridge).

[14] Hibon, P., Kashikawa, N., Willott, C. et al. 2012, ApJ, $744,89$.

[15] Longair, M. S. 1998, Galaxy Formation (Springer Berlin).

[16] Schneider, P. 2006, Extragalactic Astronomy and Cosmology (Springer Berlin Heidelberg New York).

[17] Peebles, P. J. E. 1993, Principles of Physical Cosmology (Princeton Unv. Press, Princeton New Jersey).

[18] Gradshteyn, I. S. \& Ryzhik, J. M. 1980, Table of Integrals, Series and Products (fourth edition, Academic Press, San Diego).

[19] Ouchi, M., Shimasaku, K., Furusawa, H., et al. 2010, ApJ, 723, 869.

[20] Pillepich, A., Springel, V., Nelson, D. et al. 2017, arXiv:1703.02970v2. 
[21] Behroozi, P. S., Wechsler, R. H. \& Conroy, C. 2013, ApJ, $770,57$.

[22] Gonzáles, V., Labbé, I., Bouwens, R. J. et al. 2010, ApJ, 713,115

[23] Yan, H., Yan, L., Zamojski, M. A. et al. 2011, ApJ, 728, L22.

[24] Yabe, K., Ohta, K., Iwata, I. et al. 2009, ApJ, 693 ,507.

[25] Reddy, N. A., Erb, D. K., Steidel, C. C. et al. 2005, ApJ, $633,748$.

[26] Steidel, C. C., Erb, D. K., Shapley, A. E. et al. 2010, ApJ, 717, 289.

[27] Noeske, K. G., Faber, S. M., Weiner, B. J. et al. 2007, ApJ, 660, L47.

[28] Burgarella, D., Heinis, S., Magdis, G. et al. 2011, ApJ, 734, L12.

[29] Spaans, M. \& Carollo, C. M. 1997, ApJ, 482, L93.

[30] Treu, T., Möller, P. \& Bertin, G. 2002, ApJ, 564, L13.

[31] Li, J., Wang, Q. D., Li, Z. \& Chen, Y. 2009 ApJ, 706, 693.

[32] Overzier, R. A., Heckmann, T. M., Tremonti, C. et al. 2009, ApJ, 706, 203.

[33] Oesch, P. A., Bouwens, R. J., Illingworth, G. D. et al. 2012, ApJ, 759, 135.

[34] Bouwens, R. J., Illingworth, G. D., Oesch, P. A. et al. 2012, ApJ, 754, 83.

[35] Dunlop, J. S. 2016, The Messenger, 166, 48.

[36] Zaritsky, D., Kennicutt, Jr., R.C. \& Huchra, J.B. 1994, ApJ, 420, 87.
[37] Adelberger, K. L, Steidel, C. C., Pettini, M. et al. 2005, ApJ, 619, 697.

[38] Labbé, I., Bouwens, R., Illingworth, G. D. \& Franx, M. 2006 ApJ, 649, L67.

[39] Hopkins, A. M. 2004, ApJ, 615, 209.

[40] Ciardullo, R., Gronwall, C., Wolf, C. et al. 2012, ApJ, $744,110$.

[41] Harrison, C., Alexander, D., Mullanney, M. et al. 2016, The Messenger, 163, 35.

[42] Xue, X. X., Rix, H. W., Zhao, G. et al. 2008, ApJ, 684, 1143.

[43] Jonsson, P., Cox; T. J., Primack, J. R. \& Somerville, R. S. 2006, ApJ, 637, 255.

[44] Stark, D. P., Ellis, R. S., Bunker, A. et al. 2009, ApJ, 697, 1493.

[45] Gonzáles, V., Bouwens, R. J. Illingworth, G. et al. 2014, ApJ, 781, 34.

[46] Yan, H., Dickinson, M., Giavalisco, M. et al. 2006, ApJ, 651,24 .

[47] Ryan, R. E., McCarthy, P. J., Cohen, S. H. et al. 2012, ApJ, 749, 53.

[48] Rigopoulou, D., Huang, J. S., Papovich, C. et al. 2006, ApJ, 648, 81.

[49] Reddy, N. A. \& Steidel, C. C. 2009, ApJ, 692, 778.

[50] Humphrey, P. J., Buote, D. A., O’Sullivan, E. \& Ponman, T. J. 2012, ApJ, 755, 166. 